\title{
Validation of the return of spontaneous circulation after cardiac arrest (RACA) score in two different national territories
}

\author{
Luce Caputo, Maria ; Baldi, Enrico ; Savastano, Simone ; Burkart, Roman ; Benvenuti, Claudio ; \\ Klersy, Catherine ; Cianella, Roberto ; Anselmi, Luciano ; Moccetti, Tiziano ; Mauri, Romano ; De \\ Ferrari, Gaetano M ; Auricchio, Angelo
}

\begin{abstract}
BACKGROUND The likelihood of return of spontaneous circulation (ROSC) after out-ofhospital cardiac arrest (OHCA) is influenced by unmodifiable (gender, aetiology, location, the presence of witnesses and initial rhythm) and modifiable factors (bystander CPR and the time to EMS arrival). All of these have been included in the ROSC After Cardiac Arrest (RACA) score. PURPOSE To test the ability of the RACA score to predict the probability of ROSC in two different regions with different local resuscitation networks: the Swiss Canton Ticino and the Italian Province of Pavia. METHODS AND RESULTS All OHCAs occurred between January 12015 and December 312017 were included. The original regression coefficients for all RACA score variables were applied. The probability to obtain the ROSC as measured with the RACA score was divided in tertiles. Overall, 2041 OHCAs were included in the analysis. The RACA score showed good discrimination for ROSC (AUC 0.76) and calibration, without interaction ( $\mathrm{p}$ 0.28) between the region and the probability of ROSC. The probability of ROSC was $15 \%$ for RACA scores $<0.28,20 \%$ for RACA scores between 0.28 and 0.42 , increasing to $55 \%$ for RACA scores $>0.42$. CONCLUSIONS The application of the RACA score reliably assess the probability to obtain the ROSC, with equal effectiveness in the two regions, despite different organization of the resuscitation network. Patients with a RACA score $>0.42$ had more than $50 \%$ probability to obtain ROSC.
\end{abstract}

DOI: https://doi.org/10.1016/j.resuscitation.2018.11.012

Posted at the Zurich Open Repository and Archive, University of Zurich ZORA URL: https://doi.org/10.5167/uzh-158721

Journal Article

Accepted Version

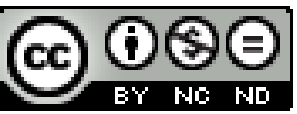

The following work is licensed under a Creative Commons: Attribution-NonCommercial-NoDerivatives 4.0 International (CC BY-NC-ND 4.0) License.

Originally published at:

Luce Caputo, Maria; Baldi, Enrico; Savastano, Simone; Burkart, Roman; Benvenuti, Claudio; Klersy, Catherine; Cianella, Roberto; Anselmi, Luciano; Moccetti, Tiziano; Mauri, Romano; De Ferrari, Gaetano M; Auricchio, Angelo (2019). Validation of the return of spontaneous circulation after cardiac arrest (RACA) score in two different national territories. Resuscitation, 134:62-68. 
DOI: https://doi.org/10.1016/j.resuscitation.2018.11.012 


\section{Accepted Manuscript}

Title: Validation of the return of spontaneous circulation after cardiac arrest (RACA) score in two different national territories

Authors: Maria Luce Caputo, Enrico Baldi, Simone Savastano, Roman Burkart, Claudio Benvenuti, Catherine

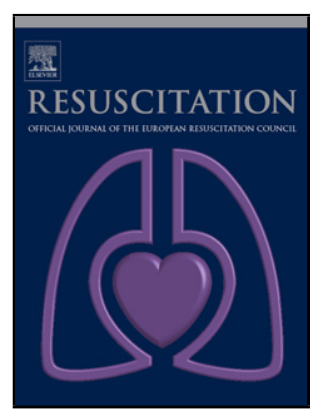

Klersy, Roberto Cianella, Luciano Anselmi, Tiziano Moccetti, Romano Mauri, Gaetano M. De Ferrari, Angelo Auricchio

PII: S0300-9572(18)31091-8

DOI: https://doi.org/10.1016/j.resuscitation.2018.11.012

Reference: RESUS 7813

To appear in:

\section{Resuscitation}

Received date:

15 July 2018

Revised date:

8 November 2018

Accepted date:

12 November 2018

Please cite this article as: Luce Caputo M, Baldi E, Savastano S, Burkart R, Benvenuti C, Klersy C, Cianella R, Anselmi L, Moccetti T, Mauri R, De Ferrari GM, Auricchio A, Validation of the return of spontaneous circulation after cardiac arrest (RACA) score in two different national territories, Resuscitation (2018), https://doi.org/10.1016/j.resuscitation.2018.11.012

This is a PDF file of an unedited manuscript that has been accepted for publication. As a service to our customers we are providing this early version of the manuscript. The manuscript will undergo copyediting, typesetting, and review of the resulting proof before it is published in its final form. Please note that during the production process errors may be discovered which could affect the content, and all legal disclaimers that apply to the journal pertain. 
Validation of the return of spontaneous circulation after cardiac arrest (RACA) score in two different national territories

Maria Luce Caputo ${ }^{1,2}$ *, M.D., Enrico Baldi ${ }^{1,3}$ *, M.D., Simone Savastano ${ }^{3}$, M.D., Roman Burkart ${ }^{4}$, C.N.S., Claudio Benvenuti ${ }^{4}$, M.B.Sc., Catherine Klersy ${ }^{5}$, M.D., Roberto Cianella ${ }^{6}$, Luciano Anselmi ${ }^{6}$, M.D., Tiziano Moccetti², M.D., Romano Mauri ${ }^{4}$, M.D., Gaetano M De Ferrari ${ }^{1,3}$, M.D., Angelo Auricchio², M.D. Ph.D

${ }^{1}$ Department of Molecular Medicine, University of Pavia, Coronary Care Unit and Cardiovascular Clinical Research Center, Fondazione IRCCS Policlinico San Matteo, Pavia, Italy; ${ }^{2}$ Cardiocentro Ticino, Lugano, Switzerland; ${ }^{3}$ Division of Cardiology, Fondazione IRCCS Policlinico San Matteo, Pavia, Italy; ${ }^{4}$ Fondazione Ticino Cuore, Breganzona, Switzerland; ${ }^{5}$ Service of Biometry and Clinical Epidemiology, Fondazione IRCCS Policlinico San Matteo, Pavia, Italy; ${ }^{6}$ Federazione Cantonale Ticinese Servizi Autoambulanze, Lugano, Switzerland.

\section{*= authors contributed equally}

Word count: 2969

\section{Corresponding author}

Caputo Maria Luce, MD

Cardiocentro Ticino, Via Tesserete 486900 Lugano, Switzerland

Tel. +41918053345; Fax +41918053173

Email: marialuce.caputo@cardiocentro.org 


\section{ABSTRACT}

\section{Background}

The likelihood of return of spontaneous circulation (ROSC) after out-of-hospital cardiac arrest (OHCA) is influenced by unmodifiable (gender, aetiology, location, the presence of witnesses and initial rhythm) and modifiable factors (bystander CPR and the time to EMS arrival). All of these have been included in the ROSC After Cardiac Arrest (RACA) score.

\section{Purpose}

To test the ability of the RACA score to predict the probability of ROSC in two different regions with different local resuscitation networks: the Swiss Canton Ticino and the Italian Province of Pavia.

\section{Methods and Results}

All OHCAs occurred between January $1^{\text {st }} 2015$ and December $31^{\text {st }} 2017$ were included. The original regression coefficients for all RACA score variables were applied. The probability to obtain the ROSC as measured with the RACA score was divided in tertiles. Overall, 2041 OHCAs were included in the analysis. The RACA score showed good discrimination for ROSC (AUC 0.76) and calibration, without interaction ( $p$ 0.28) between the region and the probability of ROSC. The probability of ROSC was $15 \%$ for RACA scores $<0.28,20 \%$ for RACA scores between 0.28 and 0.42 , increasing to $55 \%$ for RACA scores $>0.42$.

\section{Conclusions}

The application of the RACA score reliably assess the probability to obtain the ROSC, with equal effectiveness in the two regions, despite different organization of the resuscitation network. Patients with a RACA score $>0.42$ had more than $50 \%$ probability to obtain ROSC.

\section{Introduction}


The proportion of patients returning to spontaneous circulation (ROSC) after an out-ofhospital cardiac arrest (OHCA) varies considerably in Europe, and even more the 30-day survival (13). At the national level, the ROSC rate in patients with attempted cardio-pulmonary resuscitation (CPR) was as high as $50 \%$ in Cyprus and as low as $8 \%$ in Greece, and survival at discharge from hospital ranging from about $30 \%$ in Switzerland and $1 \%$ to $2 \%$ in Romania $(1,4,5)$. However, comparability of different cohorts has been questioned and direct outcome comparisons may be affected by definitions of inclusion and exclusion criteria and by local resuscitation network organization and performance (6).

In an effort to allow comparison between different EMS systems and patient cohorts, in 2011 Gräsner et al by using data from the German Resuscitation Registry developed, and then internally validated a score to predict occurrence of ROSC after OHCA, the so-called return of spontaneous circulation after cardiac arrest (RACA) (7). The RACA score considers some unmodifiable patient factors such as gender, first rhythm, and OHCA witnessed status as well as modifiable factors including cardio-pulmonary resuscitation (CPR) before EMS arrival, and the time of ambulance arrival. The authors indicated that the RACA score could contribute to preclinical quality assessment, and could help analysing the effects of different resuscitation strategies. The applicability of RACA score to other European EMS systems, first responders network, and population characteristics has been recently performed but somehow conflicting results were noted $(4,7)$. When applied to historical OHCA series recorded in the urban area of the city of Bonn (Germany), RACA score consistently overestimated observed probability of ROSC (4), whereas in study conducted in the city of Helsinki (Finland), a good overall calibration and moderate discrimination of the RACA score was observed (8). Notably, both these study were conducted in physician-staffed urban areas, a resuscitation context different from the one in which the RACA score was developed; thus, unknown is the RACA performance in mixed urban and rural areas. Finally, although RACA score allows a performance 
comparison of different EMS services in the same country, thus supporting development of strategies to improve outcome, its applicability outside German EMS services is currently unknown.

We hypothesized that RACA score would reasonably predict the probability of ROSC in a territory of two different nations, the Swiss Canton Ticino (a region in the south of Switzerland) and the Italian Province of Pavia (a province in Lombardy, Italy).

\section{METHODS}

\section{Study design and setting}

This study is a retrospective analysis of all prospectively collected OHCAs occurred between 2015 and 2017 in Swiss Canton Ticino and in Pavia's province. The Ticino Registry of Cardiac Arrest (TIRECA) has been previously described (10). The Pavia Cardiac Arrest Registry (PAVIA CARe) contains the same variables as TIRECA (11); in both registries, data are prospectively collected according to Utstein-style template (12). The study complies with the active guidelines and approved by the scientific committee of the Federazione Cantonale Ticinese Servizi Autoambulanze, and the Fondazione IRCCS Policlinico San Matteo. Furthermore, as a retrospective analysis of clinical routine data this study is in accordance with the country code of medical ethics and was approved by the local ethical competent authority.

\section{Emergency medical system and resuscitation network in Canton Ticino}

The Swiss Canton Ticino has a population of 350 '363 inhabitants (as of December $31^{\text {st }}, 2014$ ); it encompasses a territory of more than $2^{\prime} 800 \mathrm{~km}^{2}$ in the southern part of Switzerland. This region presents significant geographic challenges as the territory consists of mountains, valleys, and lakes; the population is distributed among some cities (population ranging from 5'000 to $70^{\prime} 000$ 
inhabitants) and few hundreds of rural municipalities. About $49 \%$ of the population consists of men, and overall $21 \%$ is over the age of 65 (10). An annual awareness campaign in the education of the resident population in Basic Life Support - Defibrillation (BLS-D) and to set-up a programme for wide availability of a public automatic defibrillator has been promoted by a non-profit organization Fondazione Ticino Cuore. By December $31^{\text {st }} 2017,16.4 \%$ of the resident population had completed a BLS-D course, and there were 797 public automatic external defibrillators (AEDs) in the region.

A national emergency telephone number - 144, is connected to each one of the seven regional EMS dispatching centres operating in Canton Ticino. When a cardiac arrest is suspected, a telephone assisted CPR is initiated until an ambulance arrives. The EMS dispatcher send the ambulance and, in parallel, alerts the traditional first-responders represented by police officers and fire brigade, all trained in BLS-D and equipped with an AED. If the OHCA condition is regarded as safe, the lay responders network is also activated and automatically managed by a mobile application (13). Their training includes the standard Swiss Resuscitation Council Basic Life Support (ERC BLS)/AED course for lay rescuers that complies with the recommendations of the European Resuscitation Council (14).

Each EMS service individually collects data about OHCA interventions in the registry according to Utstein methodology. The data are then reviewed periodically for quality assessment by an internal commission.

\section{EMS and resuscitation network in Pavia's Province}

The province of Pavia is a large region $(2965 \mathrm{~km} 2)$ with several rural and few urban areas with a total population of 548.722 inhabitants (as of December $31^{\text {st }}, 2014$ ). A national emergency telephone number, 118 , is connected to the regional EMS dispatching centre. The local EMS dispatcher coordinates 20 ambulances staffed with BLS-D trained personnel, and 4 ALS-trained staffed ambulances. In case of a suspected OHCA, the EMS dispatcher activates one or two 
ambulances of which at least with a physician on board and one rescuers' unit, and assists the calling bystander during chest compressions (telephone CPR).

Over the last 10 years, several initiatives have been conducted to improve public education in basic CPR and awareness campaign about the importance to use AEDs even by laypersons (14). By December 31 $1^{\text {st }} 2017,503$ PADs were available in Pavia's province $(16,17)$.

All OHCA data were consecutively and prospectively collected in the PAVIA CARe registry according to Utstein methodology, and periodically reviewed for quality assessment by an internal commission.

\section{Participants}

All consecutive OHCAs occurred in adults and collected in both the two registries since $1^{\text {st }}$ of January 2015 until $31^{\text {st }}$ of December of 2017 were considered for inclusion in the study. Patients declared dead before ambulance arrival, with a "do not resuscitate" order or with incomplete data were excluded from further analysis.

\section{Definition of return to spontaneous circulation}

As in the original paper by Gräsner et al (7), ROSC was defined as a palpable pulse for $\geq 20 \mathrm{~s}$. Failure of prehospital ROSC with ongoing CPR on admission was considered as a negative outcome (no ROSC).

\section{Statistical analysis}

All analyses were performed using Stata 15.1 (StataCorp, College Station, TX, USA). A 2-sided p<0.05 was considered statistically significant. Continuous data are reported as mean and standard deviation, median and quartiles when appropriate. Categorical data are reported as counts and percent. Data were compared between groups of patients (by national territory and by ROSC) with 
the Mann Whitney $U$ test and the Fisher exact test, respectively. The original regression coefficients for all RACA score variables (7) were applied to the combined Ticino and Pavia Registries; the probability of ROSC was calculated from this predictor index and was included as the independent variable of a logistic model for ROSC, to assess discrimination (model area under the ROC curve) and calibration (graphical assessment with the calibration belt (8) of the RACA score in our cohort. Sensitivity and specificity of the model to identify ROSC were also computed. For this purpose patients were classified as ROSC if the predicted probability was equal to or above 0.5 . The probability of ROSC was calculated for each patient using the RACA score. Then the distribution of the probability of ROSC was divided in 3 quantiles. Patients' characteristics were compared between tertiles with the Kruskall Wallis test or the Fisher exact test, as appropriate. Then a logistic model for ROSC was fitted with tertiles of the RACA score probability as the independent variables to obtain the corresponding odds ratios (OR) and $95 \%$ confidence intervals $(95 \% \mathrm{Cl})$. Model goodness of fit was assessed with the Pearson test and was always satisfied.

Finally we computed the power of showing that the observed area under the ROC curve was above 0.70 (null hypothesis).

\section{RESULTS}

During the study period, 3186 had an OHCA and were included in both the two registries. Of these, 1109 patients were declared dead before ambulance arrival or had a "do not resuscitate" order, and 36 patients (1.7\%) had incomplete dataset; all these patients were excluded, thus leaving 2041 patients (650 in Canton Ticino and 1391 in Pavia region) for subsequent analysis. 
The median age of the patients' population was 74 (IQR 61-82) years old and similar between the 2 regions (Canton Ticino: 71 [65-78] vs. Pavia: 75 [61-82] years, p 0.06). Table 1 summarizes the key demographic characteristics of both the two populations.

\section{Observed ROSC and RACA validation}

Overall, a ROSC was obtained in 581 patients (28\%) being more frequent in witnessed OHCAs, in those occurred in a public place or at work place, and having a shockable rhythm as first detected rhythm. The overall observed ROSC rate was higher in Canton Ticino than in Pavia region (38\% vs. $21 \%, p<0.001$ ) but in both cases lower than the ROSC predicted by RACA score (Ticino: $41 \%$ vs Pavia: $31 \%)$.

The RACA score model showed a good discrimination (AUC 0.76, 95\% Cl 0.74-0.78; Figure 1). Observed and predicted ROSC by RACA showed a good calibration ( $p=0.65$; Figure 1). Notably, the discrimination capacity in both regions was similar (Figure 2), without significant interaction between the region and the probability of ROSC (test for interaction $\mathrm{p} \mathrm{0.28).} \mathrm{The} \mathrm{power} \mathrm{to} \mathrm{detect} \mathrm{a} \mathrm{difference}$ of 0.06 with respect to the null hypothesis was $100 \%$. Overall RACA score reliably predicted the observed ROSC, with a specificity of $90 \%$ and a sensitivity of $39 \%$.

The tertiles of probability to obtain the ROSC are reported in Figure 3. The likelihood to observe a ROSC was $15 \%$ for RACA score values $<0.28$, increasing to $20 \%$ for RACA scores between 0.28 and 0.42 (OR 1.8, 95\% Cl 1.3-2.5, $\mathrm{P}<0.0001$ ). RACA scores of more than 0.42 showed a median probability of ROSC as high as $55 \%(\mathrm{OR} 8.3,95 \% \mathrm{Cl} 6.3-11-0, \mathrm{P}<0.0001)$. Characteristics of patients included in each of the 3 tertiles were reported in table 2 .

\section{DISCUSSION}


Our study shows a good overall calibration and discrimination of the RACA score when applied to different resuscitation networks or to different EMS services without interaction between the setting of the resuscitation and predictivity of the score. However, we found that the RACA score has a suboptimal calibration at the two extremes, i.e. in patients with the lowest or highest probability of ROSC. Although these results are encouraging for the applicability of the score in other European countries, it also suggest the need of an adjustment in resuscitation reality dealing with particularly aged population or when there is an overproportion of non-shockable rhythms as we observed.

The original purpose of RACA score intended to be a simple and generally applicable tool for predicting the initial resuscitation success adjusted to clinical conditions and information available to the EMS team on arrival at scene (7). In line with previous experiences $(9,18)$, our study confirms that RACA can reliably predict ROSC in other European countries but significantly expands previous knowledge because the external validation we did was conducted in a mixed reality of both rural and urban cohorts. Indeed, Schewe et al. (18) compared predicted RACA score to observed OHCA ROSC rates in 2070 patients occurring in the city of Bonn (Germany) whereas Kupari et al. performed an external validation of the RACA score in 681 OHCAs occurred in the metropolitan area of Helsinki, Finland (9).

The AUC reported by Gräsner et al. was 0.71 that is consistent with ours. We also noticed the absence of interaction between the region in which the resuscitation was attempted and the performance of the model. As the original model reflected the individual probability of a patient to obtain a ROSC in the German population, our observation may suggest the application of the model in different countries without adjustment of the coefficients considered in the original model. To similar conclusions arrived Kupari et al. in their work (9); indeed they found a performance of the RACA score in terms of discrimination of 0.73 , supporting the generalizability of the score. This is a 
remarkable achievement especially considering some important difference in the proportion of patients aged $\geq 80$ years and the proportion of first rhythm detected. Notably, the difference in demographic variables between our study cohort and the German Resuscitation Registry Group were similar to what recently reported by Kupari et al. (9).

In the conclusive statement of their study, Gräsner et al. said "In EMS teams operating on a high quality level, the observed ROSC rate may be higher than the predicted ROSC rate; the same should be true for a therapeutic intervention and medical treatment having positive effects. Contrarily, where the observed ROSC rate is reasonably lower than the predicted ROSC rate, further analyses of the EMS structure and process quality may be useful to identify reasons for that low performance" (7). Our study was conducted in 2 well defined EMS infrastructures which managed all OHCA cases in a large territory. In our study the observed ROSC was inferior to the expected ROSC, as predicted by RACA score. This finding is consistent with the recent study by Kupari et al. (9) but significantly differs from the single centre German study by Schewe et al. (18) or from a study comparing 7 different centres in Germany when used as one part of the EMS quality assessment in which RACA score underestimated ROSC rates (19). On the other hand and as in the Finnish experience, we noticed that RACA score is significantly lower than the observed ROSC rates, when these latter are particularly high (9). Indeed, in the $9^{\text {th }}$ and $10^{\text {th }}$ decile of probability of ROSC (values $>70 \%$ of ROSC), Kupari et al. showed a marked difference with the predicted RACA score. In our experience, in the tertile $>0.42$ we observed a probability of ROSC of at least 0.50 or higher (9). It should noticed that in the work by Schewe et al. who compared predicted and observed ROSC rates over three 5-year time period, the difference between the predicted RACA score and the observed ROSC rate progressively decreased over time (18). One may postulate that this is due to a change in the clinical characteristics of the OHCA patients but more importantly in the proportion and severity of co-mordibities that are not captured by RACA score nor by other predictive scores using survival 
at discharge and neurological outcome as clinical endpoint, such as the Cardiac Arrest Hospital Prognosis (CAPH) score (20) or the Simplified Acute Physiology Score (SAPS) (21).

To the best of our knowledge, this is the largest external validation study of the RACA score so far published that includes OHCAs occurring in both urban and rural areas at 2 European national territories. The observation that the RACA can be applied in different countries without model adjustment, potentially extends the application of the score to other European countries without further local validation.

Interestingly, most of the national RACA validation studies have used dataset referring to resuscitations attempts occurring before the year $2011(9,15,16)$. Since then, European Resuscitation Council guidelines changed. Thus, we assume that our patient cohorts better represents current cardiac arrest patient cohorts and modern post-resuscitation management of OHCA victims. In any case, should a significant discrepancy between predicted and observed ROSC rates been confirmed by other studies, the RACA-scoring system ought to be fine-tuned in order to better fit the observed ROSC rate.

\section{LIMITATIONS}

There are some limitations of our study. All patients with incomplete data about OHCA circumstances were excluded from further analysis, which implies a potential selection bias. However, only the $1.7 \%$ of all patients included in both registries had incomplete data, which can be considered negligible. All OHCAs in the two registries were classified according to the Utstein template. Considering that the RACA score equation used different categories for etiology and location respect to the Utstein template, some OHCAs in both the two registries were reclassified, accordingly, before apply the RACA score model. Thus some selection bias due to incorrect presumed aetiology or location cannot be ruled out. A potential overestimation of the ROSC probability may be determined by the inclusion 
of those patients with severe comorbidities, in who the resuscitation was precociously interrupted. However, these patients represent a small subgroup in this analysis, and were not excluded in the original RACA score validation. As in the original RACA score validation, the obtainment of a ROSC was defined by the presence of a palpable pulse for $\geq 20$ seconds. The adoption of this definition may have determined an overestimation of the ROSC rate, including also patients in who the resuscitation was only temporarily efficacious. Finally, this validation study of the RACA score was done in two European countries. The potential application of the RACA score in non-European countries needs further validation.

\section{CONCLUSIONS}

The application of the RACA score in mixed urban and rural areas is feasible to assess the probability to obtain the ROSC, with equal effectiveness in the two regions, despite different organization of the resuscitation network. Patients with a RACA score $>0.42$ had more than $50 \%$ probability to obtain ROSC. Further studies are needed to assess the applicability of the RACA score as diagnostic tool in the decision-making process of the pre-hospital cardiac arrest resuscitation.

\section{Conflicts of interest}

None.

\section{ACKNOWLEDGEMENTS}

We are grateful for all participating first responders and lay volunteers for their cooperation and support. Moreover, we want to thank all the EMS Ambulance services for their fruitful collaboration in the data collection. 


\section{REFERENCES}

1. Gräsner JT, Lefering R, Koster RW, Masterson S, Böttiger BW, Herlitz J, et al; EuReCa ONE Collaborators. EuReCa ONE-27 Nations, ONE Europe, ONE Registry: A prospective one month analysis of out-of-hospital cardiac arrest outcomes in 27 countries in Europe. Resuscitation. 2016;105:188-95.

2. Berdowski J, Berg RA, Tijssen JG, Koster RW. Global incidences of out-ofhospital cardiac arrest and survival rates: systematic review of 67 prospective studies. Resuscitation. 2010;81:1479_ 87.

3. Herlitz J, Bång A, Gunnarsson J, Engdahl J, Karlson BW, Lindqvist J, et al. Factors associated with survival to hospital discharge among patients hospitalised alive after out of hospital cardiac arrest: change in outcome over 20 years in the community of Göteborg, Sweden. Heart. 2003;89:25-30.

4. Fischer M, Fischer NJ, Schuttler J. One-year survival after out-of-hospital cardiac arrest in Bonn city: outcome report according to the Utstein style. Resuscitation. 1997;33:233-43.

5. Holler NG, Mantoni T, Nielsen SL, Lippert F, Rasmussen LS. Long term survival after out-ofhospital cardiac arrest. Resuscitation. 2007;75:23-8.

6. Martinell L, Nielsen N, Herlitz J, Karlsson T, Horn J, Wise MP, et al. Early predictors of poor outcome after out-of-hospital cardiac arrest. Crit Care. 2017;21:96.

7. Gräsner JT, Meybohm P, Lefering R, Wnent J, Bahr J, Messelken M, et al; German Resuscitation Registry Study Group. ROSC after cardiac arrest--the RACA score to predict outcome after out-of-hospital cardiac arrest. Eur Heart J. 2011 Jul;32(13):1649-56.

8. Nattino, G, Finazzi S, Bertolini G. A new test and graphical tool to assess the goodness of fit of logistic regression models. Statist. Med. 2016; 35: 709-720. 
9. Kupari P, Skrifvars M, Kuisma M. External validation of the ROSC after cardiac arrest (RACA) score in a physician staffed emergency medical service system. Scand J Trauma Resusc Emerg Med. 2017;25:34.

10. Mauri R, Burkart R, Benvenuti C, Caputo ML, Moccetti T, Del Bufalo A et al. Better management of out-of-hospital cardiac arrest increases survival rate and improves neurological outcome in the Swiss Canton Ticino. Europace. 2016;18:398-404.

11. Savastano S, De Servi S, Oltrona Visconti L, Raimondi M; all the Pavia CARE researchers. The Pavia Cardiac Arrest REgistry - Pavia CARE. It's time for Cardiologists to be engaged in cardiac arrest. Int J Cardiol. 2015;185:93-4.

12. Perkins GD, Jacobs IG, Nadkarni VM, Berg RA, Bhanji F, Biarent D, et al; Utstein Collaborators. Cardiac arrest and cardiopulmonary resuscitation outcome reports: update of the Utstein Resuscitation Registry Templates for Out-of-Hospital Cardiac Arrest: a statement for healthcare professionals from a task force of the International Liaison Committee on Resuscitation (American Heart Association, European Resuscitation Council, Australian and New Zealand Council on Resuscitation, Heart and Stroke Foundation of Canada, InterAmerican Heart Foundation, Resuscitation Council of Southern Africa, Resuscitation Council of Asia); and the American Heart Association Emergency Cardiovascular Care Committee and the Council on Cardiopulmonary, Critical Care, Perioperative and Resuscitation. Circulation. 2015; 132:1286-300.

13. Caputo ML, Muschietti S, Burkart R, Benvenuti C, Conte G, Regoli F, et al. Lay persons alerted by mobile application system initiate earlier cardio-pulmonary resuscitation: A comparison with SMS-based system notification. Resuscitation. 2017;114:73-78. 
14. Monsieurs KG, Nolan JP, Bossaert LL, Greif R, Maconochie IK, Nikolaou NI, et al; ERC Guidelines 2015 Writing Group. European Resuscitation Council Guidelines for Resuscitation 2015: Section 1. Executive summary. Resuscitation. 2015;95:1-80.

15. Hasselqvist-Ax I, Riva G, Herlitz J, Rosenqvist M, Hollenberg J, Nordberg P, et al. Early cardiopulmonary resuscitation in out-of-hospital cardiac arrest. N Engl J Med. 2015;372:230715.

16. https://www.areu.lombardia.it/web/home/mappa-dae-lombardia

17. Baldi E, Savastano S. AED use before EMS arrival: When survival becomes a matter of law and system in Italy, which can be improved. Eur Heart J. 2018;39:1664.

18. Schewe JC, Kappler J, Heister U, Weber SU, Diepenseifen CJ, Frings B, et al. Outcome of outof-hospital cardiac arrest over a period of 15 years in comparison to the RACA score in a physician staffed urban emergency medical service in Germany. Resuscitation. 2015; 96:232238.

19. Neukamm J, Gräsner JT, Schewe JC, Breil M, Bahr J, Heister U, et al. The impact of response time reliability on CPR incidence and resuscitation success: a benchmark study from the German Resuscitation Registry. Crit Care. 2011;15: R282.

20. Maupain C, Bougouin W, Lamhaut L, Deye N, Dieh I L, Geri G, et al. The CAHP (Cardiac Arrest Hospital Prognosis) score: a tool for risk stratification after out-of-hospital cardiac arrest. Eur Heart J. 2016;37:3222-3228.

21. Bisbal M, Jouve E, Papazian L, de Bourmont S, Perrin G, Eon B, et al. Effectiveness of SAPS III to predict hospital mortality for post-cardiac arrest patients. Resuscitation. 2014;85:939-944.

\section{FIGURES LEGEND}

Figure 1. Left panel: Receiver Operating Characteristic (ROC) curve of RACA score; the area under the curve of 0.76 corresponds to a good discrimination of the model in the overall population. Right 
panel: Calibration curve for the model in the overall population. The bisecting line corresponds to perfect calibration of the model (perfect agreement between observed deaths and predicted deaths. The line is entirely included in the shaded area corresponding to the $80 \%$ and $95 \%$ confidence intervals for the observed-predicted relationship, denoting that the model is well calibrated (there is neither over nor underestimation of the mortality).

Figure 2. Receiver Operating Characteristic (ROC) curve of RACA score in Canton Ticino (blu line) and in Pavia's Province (red line).

Figure 3. Tertiles of probability of ROSC by RACA score values.

Figure 1.
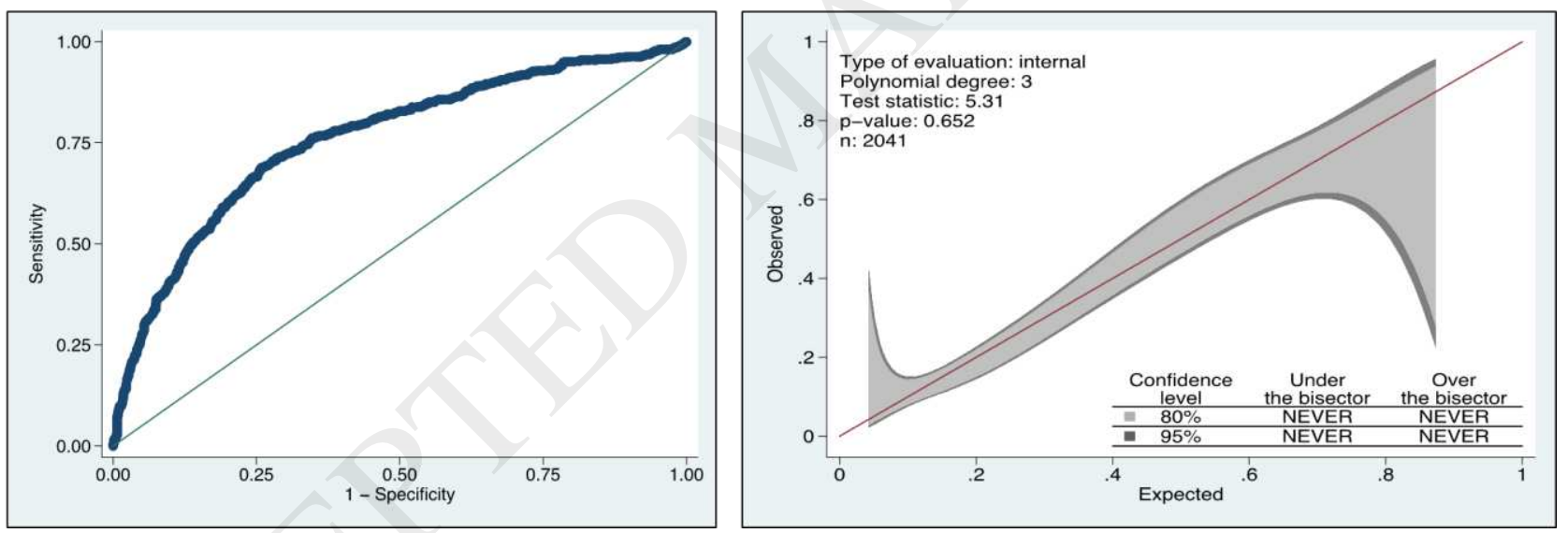
Figure 2.

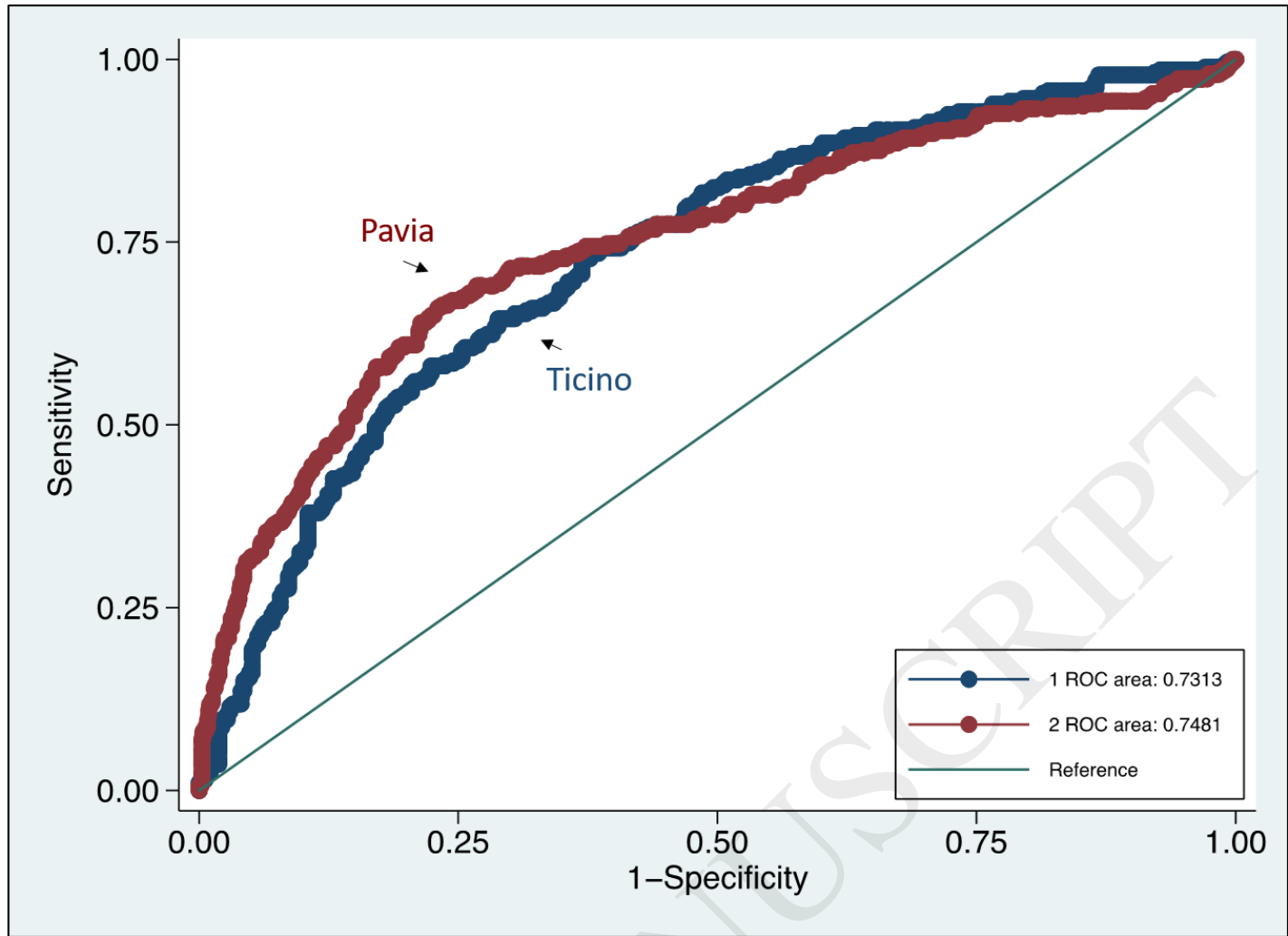

Figure 3.

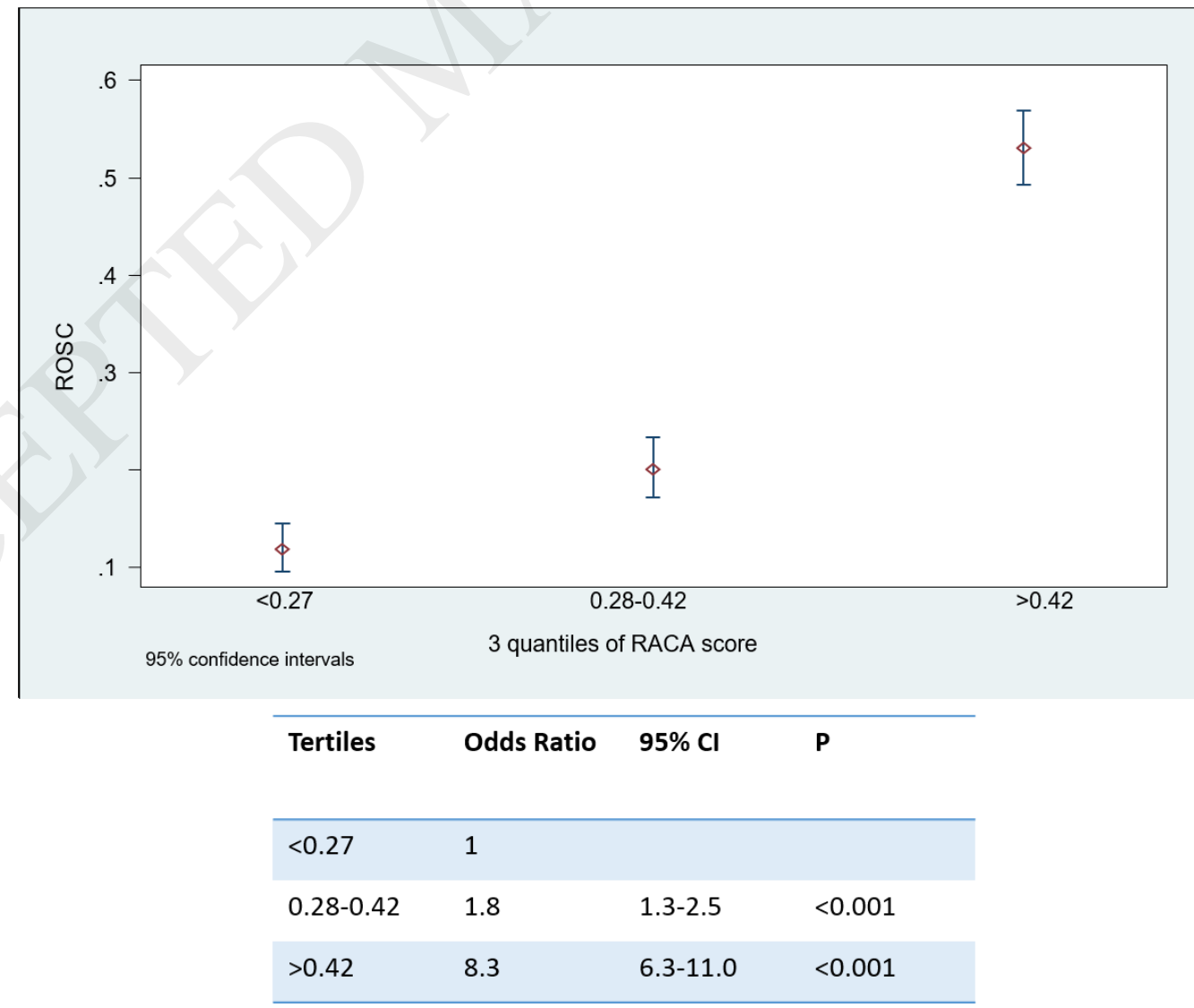


Table 1. Demographic characteristics according to RACA score variables.

\begin{tabular}{|c|c|c|c|c|}
\hline Variable & All $(n=2041)$ & Ticino $(n=650)$ & Pavia (n=1391) & $P$ value \\
\hline Male gender, n (\%) & $1280(62)$ & $439(67)$ & $841(60)$ & 0.005 \\
\hline Age, median (IQR) & $74(61-82)$ & $71(65-78)$ & $75(61-82)$ & 0.06 \\
\hline Age $>80$ years old, $n(\%)$ & $808(39)$ & $205(31)$ & $603(43)$ & $<0.001$ \\
\hline Etiology, $\mathrm{n}(\%)$ & & & 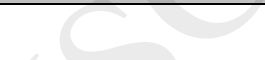 & 0.001 \\
\hline Cardiac & $1780(86)$ & $488(75)$ & $1292(91)$ & \\
\hline Trauma & $85(4)$ & $23(3)$ & $62(5)$ & \\
\hline Respiratory & $97(5)$ & $69(10)$ & $28(2)$ & \\
\hline Intoxication & $51(3)$ & $45(7)$ & $6(1)$ & \\
\hline Other/unknown & $35(2)$ & $32(5)$ & $3(1)$ & \\
\hline Witness, n (\%) & & & & 0.010 \\
\hline None & $573(27)$ & $206(31)$ & $367(26)$ & \\
\hline Lay people & $1112(53)$ & $317(47)$ & $795(56)$ & \\
\hline Professionals & $352(20)$ & $132(22)$ & $220(15)$ & \\
\hline Location, $\mathrm{n}(\%)$ & & & & 0.001 \\
\hline At home & $1524(75)$ & $421(65)$ & $1103(79)$ & \\
\hline
\end{tabular}

support; EMS: Emergency medical system; EMS: emergency medical service. 


\begin{tabular}{|l|l|l|l|l|}
\hline Nursing home & $153(7)$ & $33(5)$ & $120(8)$ & \\
\hline Work place & $26(1)$ & $11(2)$ & $15(1)$ & \\
\hline Doctor's office & $17(1)$ & $17(2)$ & $0(0)$ & \\
\hline Public place & $275(13)$ & $128(19)$ & $147(11)$ & \\
\hline other & $53(3)$ & $47(7)$ & $6(1)$ & \\
\hline Rhythm, N (\%) & & & & \\
\hline Shockable & & & & \\
\hline Asystole & $408(20)$ & $149(23)$ & $259(19)$ & \\
\hline Pulseless activity & & & & \\
\hline other & $931(45)$ & $266(40)$ & $665(48)$ & \\
\hline Bystander BLS, N (\%) & $925(45)$ & $458(70)$ & $467(34)$ & \\
\hline & & & & \\
\hline & $10.4(7.9-14.0)$ & $10.1(7.6-13.5)$ & $10.6(8.0-14.0)$ & 0.05 \\
\hline & & $207(31)$ & $359(26)$ & \\
\hline
\end{tabular}

Table 2. Patients' characteristics according to tertiles of the probability of ROSC derived with the RACA score.

\begin{tabular}{|l|l|l|l|}
\hline Variable & $\begin{array}{l}\text { Probability of } \\
\text { ROsC }\end{array}$ & $\begin{array}{l}\text { Probability of } \\
\text { ROSC }\end{array}$ & $\begin{array}{l}\text { Probability of } \\
\text { ROSC }\end{array}$ \\
& $<0.27$ & $\mathbf{0 . 2 7 - 0 . 4 2}$ & $>0.42$ \\
$\mathrm{~N}=689$ & $\mathrm{~N}=677$ & $\mathrm{~N}=682$ \\
\hline Male gender, $\mathbf{n}(\%)$ & $429(62)$ & $402(60)$ & $449(66)$ \\
\hline Age>80 years old, $\mathbf{n}(\%)$ & $353(51)$ & $289(43)$ & $166(24)$ \\
\hline
\end{tabular}




\begin{tabular}{|c|c|c|c|}
\hline \multicolumn{4}{|l|}{ Etiology, n (\%) } \\
\hline Cardiac & $629(91)$ & $611(90)$ & $540(79)$ \\
\hline Trauma & $57(8)$ & $23(3)$ & $5(1)$ \\
\hline Respiratory & $0(0)$ & $16(2)$ & $81(12)$ \\
\hline Intoxication & $0(0)$ & $7(2)$ & $44(6)$ \\
\hline Other/unknown & $3(1)$ & $20(3)$ & $12(2)$ \\
\hline \multicolumn{4}{|l|}{ Witness, n (\%) } \\
\hline None & 337 (49) & $163(24)$ & $74(11)$ \\
\hline Lay people & $204(30)$ & $414(61)$ & $497(73)$ \\
\hline Professionals & $145(21)$ & $99(15)$ & $111(16)$ \\
\hline \multicolumn{4}{|l|}{ Location, n (\%) } \\
\hline At home & $561(81)$ & $549(81)$ & $414(61)$ \\
\hline Nursing home & $68(10)$ & $51(7)$ & $34(5)$ \\
\hline Work place & $6(1)$ & $6(2)$ & $14(2)$ \\
\hline Doctor's office & $0(0)$ & $0(0)$ & $17(2)$ \\
\hline Public place & $53(7)$ & $62(9)$ & $160(24)$ \\
\hline other & $1(1)$ & $9(1)$ & $43(6)$ \\
\hline \multicolumn{4}{|l|}{ Rhythm, N (\%) } \\
\hline Shockable & $4(1)$ & $67(16)$ & $337(83)$ \\
\hline
\end{tabular}




\begin{tabular}{|l|l|l|l|}
\hline Asystole & $508(73)$ & $333(49)$ & $90(13)$ \\
\hline Pulseless activity & $172(25)$ & $237(35)$ & $157(23)$ \\
\hline other & $5(3)$ & $40(28)$ & $98(68)$ \\
\hline Bystander BLS, N (\%) & $124(18)$ & $327(48)$ & $474(69)$ \\
\hline Time to EMS arrival, min (IQR) & $12.0(9.0-15.0)$ & $10.2(7.8-13.6)$ & $9.3(7.0-12.4)$ \\
\hline Survival at discharge, N (\%) & $10(2)$ & $57(8)$ & $183(27)$ \\
\hline
\end{tabular}

BLS: Basic life support; EMS: Emergency medical system; EMS: emergency medical service. 